\title{
An Efficient Solid-Phase Extraction-Based Liquid Chromatography Method to Simultaneously Determine Diastereomers $\alpha$-Tocopherol, Other Tocols, and Retinol Isomers in Infant Formula
}

\author{
Baifen Huang, Zengxuan Cai, Jingshun Zhang, and Jiaojiao Xu $\mathbb{D}$ \\ Department of Physicochemical \& Toxicology, Zhejiang Provincial Center for Disease Control and Prevention, \\ Hangzhou 310051, China \\ Correspondence should be addressed to Jiaojiao Xu; jjxu@cdc.zj.cn
}

Received 4 February 2021; Revised 5 March 2021; Accepted 11 March 2021; Published 20 March 2021

Academic Editor: Alessandra Durazzo

Copyright ( 2021 Baifen Huang et al. This is an open access article distributed under the Creative Commons Attribution License, which permits unrestricted use, distribution, and reproduction in any medium, provided the original work is properly cited.

\begin{abstract}
The separation and simultaneous quantitation of diastereomers of DL- $\alpha$-tocopherol, eight tocol forms, and retinols (trans and cis) have been conducted by reversed-phase liquid chromatography followed by solid-phase extraction. A chiral silica stationary phase modified with polysaccharide derivative on the monodisperse macroporous silica gel (Unichiral OD-5H column, $150 \mathrm{~mm} \times 4.6 \mathrm{~mm}, 5 \mu \mathrm{m}$, NanoMicro Technology Co., Ltd.) was employed for eluting each target compound. Instead of conventional solvent extract, a green and eco-friendly solid-phase extraction column, packing with nonpolar polystyrene divinylbenzene, was optimized in terms of capacity and solvent used in steps. Validation of the method was examined and confirmed to be satisfactory, with excellent linearity regression $(r>0.9999)$, acceptable accuracy $(74.66 \% \sim 112.92 \%)$, and precision $(0.20 \% \sim 10.52 \%)$ results. Limit of detection ranged from $0.05 \mathrm{mg} \cdot \mathrm{kg}^{-1}$ (retinols) to $0.4 \mathrm{mg} \cdot \mathrm{kg}^{-1}$ (tocols). The method was checked by infant formula reference material SRM 1849a as well, which illustrated good agreement of mass fraction with certified value and enriched the important isomer data.
\end{abstract}

\section{Introduction}

Measurement of vitamins in foods and supplements is important for monitoring and controlling nutrient intakes of various populations, especially for specific groups (like elders and infants). Excess and deficient intakes of fat-soluble vitamins could cause a disorder of protein metabolism [1], immune system, version and regulation of cell growth, and differentiation [2].

Vitamin A belongs to the fat-soluble vitamin group that helps maintain normal reproduction, vision, and immune function. It comes in several forms (like retinol, retinal, retinoic acid, or retinyl ester). Isomers of vitamin A have different activities. All-trans-retinol is defined to the $100 \%$ reference activity level, while 13-cis-retinol and 11-cis are $75 \%$ and $30 \%$ active, respectively, and the other isomers have activities lower than 20\% [3]. In general, all-trans and 13-cis retinol are the most common forms found in foods and supplements. However, the determinations of vitamin A always focus on total vitamin A or total retinol only, which could lead to underestimation of vitamin A when cis-isomers are also present.

Vitamin E, another main group of fat-soluble vitamins, plays an important role in animal reproduction, antioxidant, and anticancer activities. Consisting of tocopherols and tocotrienols $(\alpha-, \beta-, \gamma$-, and $\delta$-form), both natural and synthetic forms of vitamin $\mathrm{E}$ are used as additives in food and food supplement. For the sake of different presentence of 2, 4', and 8' asymmetric carbon atoms in tocopherol molecule, the natural $\alpha$-tocopherol (D- $\alpha$-tocopherol) and synthetic tocopherol (DL- $\alpha$-tocopherol) result in eight stereoisomers $[4,5]$. Natural D- $\alpha$-tocopherol is the most effective assigned 1.49 IU vitamin $\mathrm{E}$ equivalent, whereas DL- $\alpha$-tocopherol (all-rac) was assigned 1.10 IU vitamin E 
equivalent [6]. Besides, $\beta$-, $\gamma$-, $\delta$-tocopherol and tocotrienol congeners act out significantly different activities. Consequently, the distinction of $\alpha$-tocopherol forms and vitamin $\mathrm{E}$ isomers is important for quality control and analysis.

Liquid chromatography is the method most frequently employed for the analysis of retinol and tocol isomers. Normal-phase liquid chromatography (NP-LC) has successfully been applied to separations of isomers of retinol [7-9], and tocol isomers, which has been reviewed by Ruperez et al. and Fanali et al. [5, 10]. When saponification is not essential, the NP-LC method could conduct direct quantitative of target compounds through sample extraction and elution with hexane. However, considering robustness of the chromatographic columns, reproducibility of chromatographic peak characteristics, and reduction of volatile and hazardous solvents, reverse-phase liquid chromatography (RP-LC) offers greater suitability, especially in the aspect of multiple vitamin isomers separation. Silica-C30, pentafluorophenyl (PFP), and high-density C18 stationary phase with polymeric stationary phase have been employed in RP-LC for the separation of $\beta$ - and $\gamma$-tocols $[4,11,12]$, while it has been a bit rarely used for retinol isomers.

To distinguish the natural tocopherol (D- $\alpha$-tocopherol) in a product, it is only necessary to demonstrate a single peak using the chiral stationary column. To date, several publications have been reported to separate $\alpha$-tocopherol stereoisomers, based on the three chiral centers in the phytyl tail. With different polymeric bonding modified chiral stationary phases, some scientific researches have been done to separate isomers of DL- $\alpha$-tocopherol into more than two peaks $[4,13,14]$. Although there is rarely a report showing the differentiation between diastereomers of vitamin $\mathrm{E}$ and retinol congeners simultaneously, the differentiation by RPLC would be usefully considered for the sake of reversedphase mode advantages and versatilities.

Solvent extraction is a classical method in vitamin A and vitamin $\mathrm{E}$ analysis, as in the case of the standardized method in authority [15-17]. Except for tedious steps, those methods are not satisfied with the economy and environmental friend. For their peculiarities, extraction methods, including solid-phase extraction (SPE), supercritical fluid extraction, and pressurized liquid extraction, have been developed to meet the scientific trend of simplification, speediness, wastage reduction, costs, and safety. Among them, SPE is a rapid, effective, and versatile technique and has been employed in various matrices for fat-soluble compounds extraction, such as the concentration of tocols in rice brans [18], and tocols and carotenoids in cereal samples [19]. To the best of our knowledge, there is no study to describe simultaneous extraction of vitamin $\mathrm{E}$ congeners and retinols (cis and trans isomers) in infant formula by SPE.

The present study aims to develop and validate an accurate, precious, sensitive, and eco-friendly RP-LC method for the determination of tocols (tocopherols and tocotrienols) and retinol (cis and trans) isomers simultaneously using polysaccharide derivative modified silica stationary phase (Unichiral OD-5H column, $150 \mathrm{~mm} \times 4.6 \mathrm{~mm}, 5 \mu \mathrm{m}$ ), which was proven to be sufficient for the distinction of the DL- $\alpha$-tocopherol and D- $\alpha$-tocopherol. A green sample preparation technique was employed instead of solvent extraction and was applied in infant formula samples successfully.

\section{Materials and Methods}

2.1. Chemicals and Materials. All-trans-retinol, 9-cis-retinol, and 13-cis-retinol were obtained from Toronto Research Chemicals (Irvine, CA, USA). D-Tocopherols (D- $\alpha-, \beta-, \gamma^{-}$, and $\delta$-tocopherol) and tocotrienols (D- $\alpha-, \beta-, \gamma-$, and $\delta$-tocotrienol) were obtained from Supelco (Bellefonte, PA, USA), as well as DL- $\alpha$-tocopherol. Stock solutions $\left(1 \mathrm{mg} \cdot \mathrm{mL}^{-1}\right.$ of all-trans-retinol, $100 \mu \mathrm{g} \cdot \mathrm{mL}^{-1}$ of cis-retinol, $20 \mathrm{mg} \cdot \mathrm{mL}^{-1}$ of DL- $\alpha$-tocopherol, $5 \mathrm{mg} \cdot \mathrm{mL}^{-1}$ of tocopherols and tocotrienols) were prepared in anhydrous alcohol and stored in brown glass bottles at $-20^{\circ} \mathrm{C}$. Their concentrations were evaluated spectrophotometrically based on their specific absorption coefficients: $\alpha$-tocopherol $=75.8$ at $292 \mathrm{~nm}$, $\beta$-tocopherol $=89.4$ at $296 \mathrm{~nm}, \quad \gamma$-tocopherol $=91.4$ at $298 \mathrm{~nm}, \delta$-tocopherol $=87.3$ at $298 \mathrm{~nm}, \alpha$-tocotrienol $=91.0$ at $\quad 292 \mathrm{~nm}, \quad \beta$-tocotrienol $=87.5 \quad$ at $\quad 295 \mathrm{~nm}$, $\gamma$-tocotrienol $=103.0$ at $298 \mathrm{~nm}, \delta$-tocotrienol $=83.0$ at $292 \mathrm{~nm}$, all-trans-retinol $=1830$ at $325 \mathrm{~nm}$, and 13-cis-reti$\mathrm{nol}=1686$ at $328 \mathrm{~nm}[15,20]$. Take one milliliter of each stock standard solution and makeup to $100 \mathrm{~mL}$ with methanol in a $100-\mathrm{mL}$ volumetric flask. Working solutions were prepared by methanol in available dilution times.

HPLC grade of methanol $(\mathrm{MeOH})$ and acetonitrile (ACN) were purchased from Merck (Darmstadt, Germany). Ultrapure water was obtained from Millipore (Bedford, MA, USA). All other reagents were of analytical grade and were purchased from local suppliers. The packings with polystyrene divinylbenzene (PS-DVB) SPE cartridges in three brands were evaluated, SelectCore PSN from NanoMicro Technology Co., Ltd. (Suzhou, China), Bond Elut Plexa from Agilent Technologies, Inc. (CA, USA), and Welchrom PS/ DVB column from Welch Technologies Shanghai Co., Ltd. (Shanghai, China). The infant powder matrix sample was purchased and information was collected from a local supplier.

2.2. Instrumentation. LC system was composed of I-Class Waters Acquity ${ }^{\mathrm{TM}}$ UPLC with a fluorescent detector (Large volume flow cell), a photo-diode array detector, and a 20 $\mu \mathrm{L}$ sample loop. The chiral column, $150 \mathrm{~mm} \times 4.6 \mathrm{~mm}$ inner diameter, $5 \mu \mathrm{m}$ particle size, Unichiral OD-5H column (NanoMicro Technology Co., Ltd, China), which was packed with polysaccharide derivative on the surface of spherical silica stationary phase, was conducted at $35^{\circ} \mathrm{C}$. The two-component mobile phase (A-water, B-75\% ACN/ $25 \% \mathrm{MeOH}$ ) was delivered at a flow rate of $1.2 \mathrm{~mL} \cdot \mathrm{min}^{-1}$ as the following time table: $0-23 \mathrm{~min}, 75 \% \mathrm{~B} ; 23-32.5 \mathrm{~min}$, 75\% 80\% B; 32.5-35 min, 80\% 100\% B; 35-39 min, $100 \%$ $\mathrm{B}, 39-39.5 \mathrm{~min}, 100 \% \sim 75 \% \mathrm{~B}$; total run time was $45 \mathrm{~min}$. Tocopherols and tocotrienols were detected with fluorescence at $294 \mathrm{~nm}$ excitation and $328 \mathrm{~nm}$ emission, while retinols were detected with a photo-diode array detector at $328 \mathrm{~nm}$. 
2.3. Method Validation. The established RP-LC method was validated in an aspect of specificity, linearity, range, limits of detection (LOD), limits of quantification (LOQ), precision, and accuracy. The linearity of each analyte was evaluated by calculating the slope, intercept, and correlation coefficient of each calibration curve. The LODs and LOQs were determined by spiking various low concentration levels and determined as the lowest concentrations that produce chromatographic peaks at a signal-to-noise ratio $(\mathrm{S} / \mathrm{N})$ of 3 and 10 , respectively. Accuracy and precision of the method were conducted by adding three levels of standard working solution to infant formula sample in six parallel levels, whereas spiking concentrations were based $0.75-, 1.5-$, and 3-folds on the content of analytes in infant formula sample (which is mainly calculated based on the content of D- $\alpha$-tocopherol). The selectivity of the method was analyzed by comparing the chromatograms of analyte-free samples and the spiked ones. Furthermore, the method was validated and applied for an infant/adult nutritional formula SRM 1849a of reference material supplied by the National Institute of Standards and Technology (NIST).

2.4. Sample Preparation. According to significantly different uniformity of dry and wet blended powder samples, sample homogenization was conducted as follows: transfer $20 \mathrm{~g}$ of dry blended/nonhomogeneous infant formula powder samples, accurately weighed, to a $250-\mathrm{mL}$ bottle. Dissolve in warm water (about $50-60^{\circ} \mathrm{C}$ ) until no obvious granule, cool down, and make up to $100 \mathrm{~g}$ with water. Transfer $5 \mathrm{~g}$ reconstituted sample to a screw-top centrifuge tube. For wet blended/homogeneous powder samples, transfer $1.0 \mathrm{~g}$ to a screw-top $50 \mathrm{~mL}$ centrifuge tube. Add $5 \mathrm{~mL}$ warm water of approximately $50^{\circ} \mathrm{C}$ and shake to dissolve [21].

For extraction, samples were submitted to a modified saponification procedure as described in mandate standardized method [17], with $0.2 \mathrm{~g}$ ascorbic acid, $6 \mathrm{~mL}$ ethanol containing $0.1 \%$ butylated hydroxytoluene (BHT), and $3 \mathrm{~mL}$ $50 \%$ potassium hydroxide for $30 \mathrm{~min}$ at $80^{\circ} \mathrm{C}$ constant temperature oscillation water bath. The tube was placed in an ice bath to cool down. Then onefold saponification solution bulk of the water was added. The test tube was centrifuged and the supernatants were loaded to SPE cartridge. To avoid the destruction of labile vitamins, all saponification work was carried out under subdued light.

The total of the above supernatants was passed through PS-DVB cartridges, which were conditioned with $3 \mathrm{~mL}$ of methanol and $3 \mathrm{~mL}$ of water. After washing with $5 \mathrm{~mL}$ of $10 \%$ aqueous methanol solution repeated twice, retained constituents were eluted with $7.5 \mathrm{~mL}$ of $\mathrm{ACN} / \mathrm{MeOH}$ mixture $(75 / 25, v / v)$. Making up to $10 \mathrm{~mL}$ by water, the lotions were filtered and injected into the HPLC column. All measurements were performed in triplicate. The results of all measurements are expressed as means $\pm \mathrm{SD}$.

\section{Results and Discussion}

3.1. Separation of Asymmetric $\alpha$-Tocopherol and Retinol Isomers. The first and crucial study was carried out here to pick up the analytical column and address the optimization of the mobile phase. Different stationary phase columns were considered, as alkyl-bonded C30 silica, high-density C18 stationary phase with polymeric bonding, and PFP column were reported to separate $\beta$ and $\gamma$ tocol isomers. In an aspect of retinols, few pieces of literature were discussed about the RP-LC method for trans and cis isomers separation. In the present study, C30 and PFP stationary phases were proven to be of satisfying performance for the separation of trans and cis retinols, while both of them could not distinguish DL- $\alpha$-tocopherol and D- $\alpha$-tocopherol. Normal C18 stationary phase could not be used for the separation of $\beta$ and $\gamma$ tocol isomers. According to previous reports, chiral stationary phases were available for asymmetric $\alpha$-tocopherol. In this study, a chiral silica stationary phase modified with polysaccharide derivative on the monodisperse macroporous silica gel (Unichiral OD-5H column, $150 \mathrm{~mm} \times 4.6 \mathrm{~mm}, 5 \mu \mathrm{m})$ was tested. Methanol and acetonitrile were primarily examined as mobile phases. Starting isocratic elution with methanol, the overlapping peak of $\delta$-tocopherol and $\gamma$-tocotrienol was observed, as well as the longer retention times of all analytes. With acetonitrile solvent, the elution was so quick that the complete separation of trans and cis retinols could not be achieved. Different proportions of these two solvents were tested consequently. The best separation was conducted by the gradient elution system started with $75 \%$ of $\mathrm{ACN} / \mathrm{MeOH}$ (75/25, v/v) mixture. Under optimization conditions, those compounds were separated sufficiently by the Unichiral OD$5 \mathrm{H}$ column and the whole elution lasted less than $40 \mathrm{~min}$. Figure 1(c) illustrates that there would be two peaks in DL$\alpha$-tocopherol standard solution, while it would be only one peak in the D- $\alpha$-tocopherol standard, which would be employed to distinguish the $\alpha$-tocopherol form in samples.

3.2. Optimization of SPE Parameters. For disadvantages of solvent extraction in long extraction time with a lot of toxic solvents consumption and tedious steps, several environmental extraction methods have been developed for the release of tocols depending on the characteristics of the samples. In this study, a green SPE method was established and optimized, including the choice of sorbent, wash, and eluent solvent.

The simultaneous extraction of tocols and retinols was more complicated. A commercial nonpolar polystyrene divinylbenzene (PS-DVB) packing column was taken on researchers' interest based on its advantage of highthroughput assays, alkali resistance, and strong hydrophobicity. Bond Elut Plexa column $(500 \mathrm{mg}, 6 \mathrm{~mL})$, Bond Elut Plexa column ( $200 \mathrm{mg}, 6 \mathrm{~mL}$ ), SelectcCore PSN column (200 mg, $6 \mathrm{~mL}$ ), and Welchrom PS/DVB column (200 mg, $6 \mathrm{~mL}$ ) were compared and illustrated in Figure 2. The simulated saponification extract solution consists of available tocols and retinols standards mixture, and $40 \%$ potassium hydroxide in ethanol solution. Figure 2 illustrates that different brand packing columns showed different retention capabilities for analytes. There was no significant difference between the capabilities of SelectcCore PSN and that of Welchrom PS/DVB. Bond Elut Plexa columns demonstrated fewer capacities for partial analytes, especially 

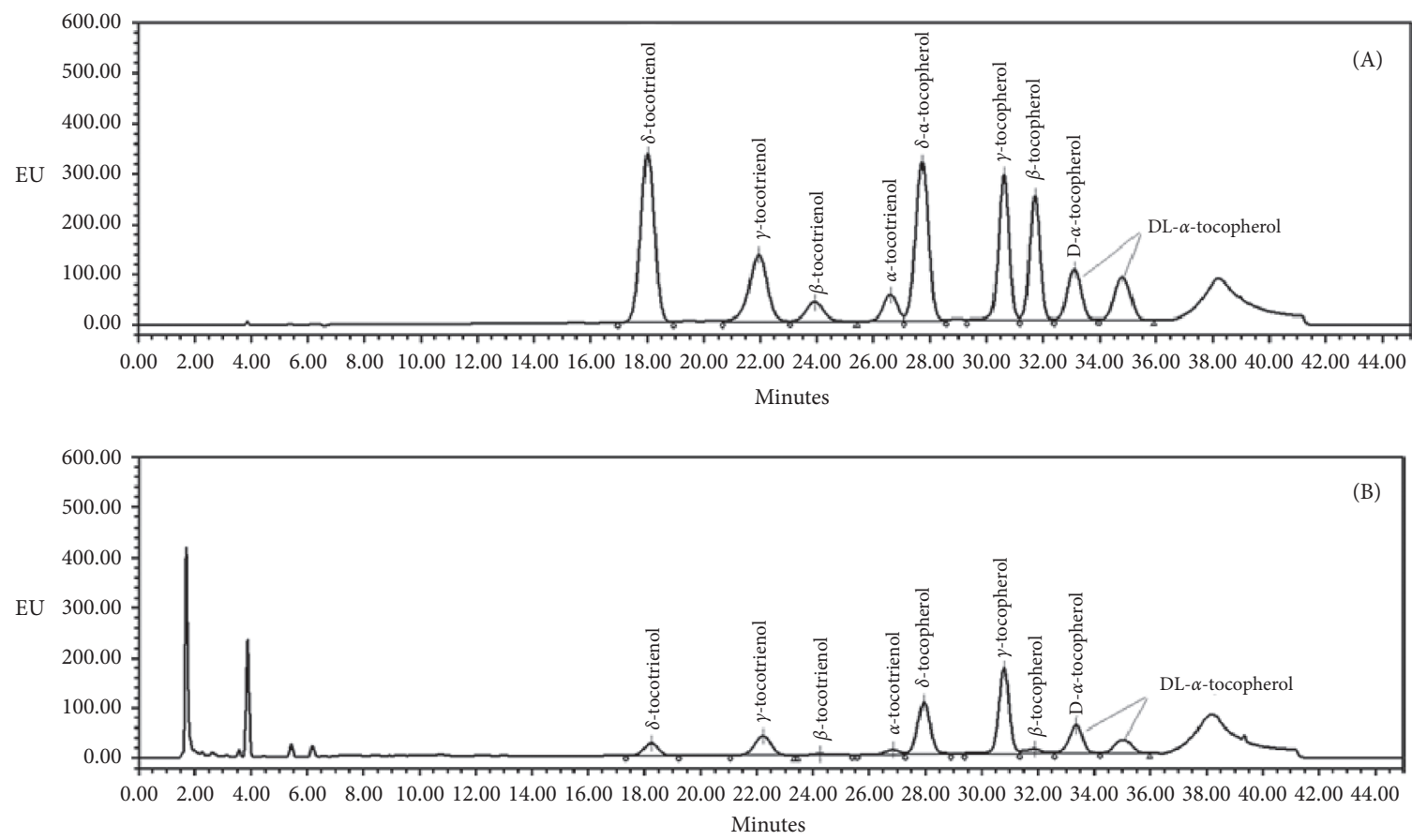

(a)
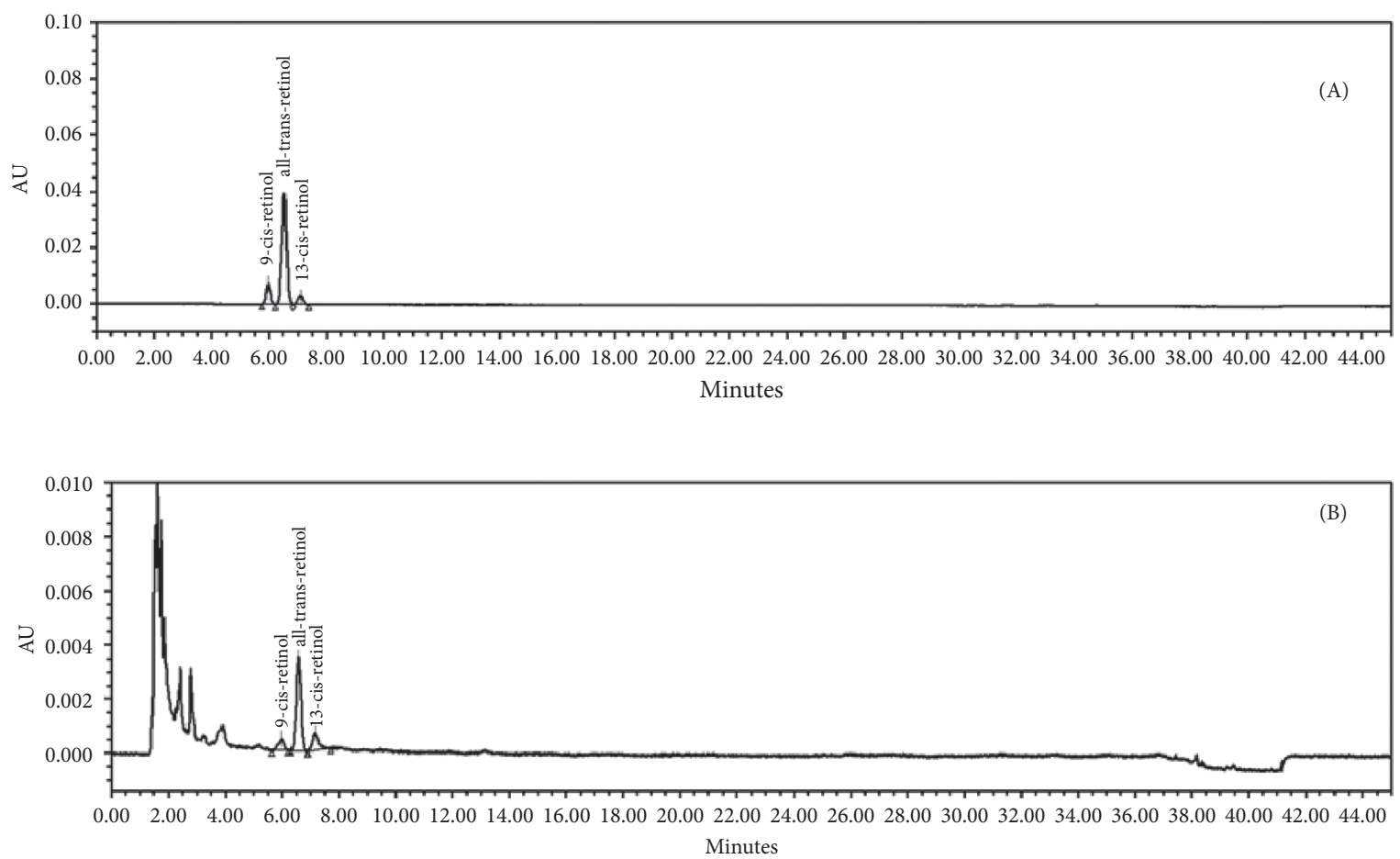

(b)

Figure 1: Continued. 

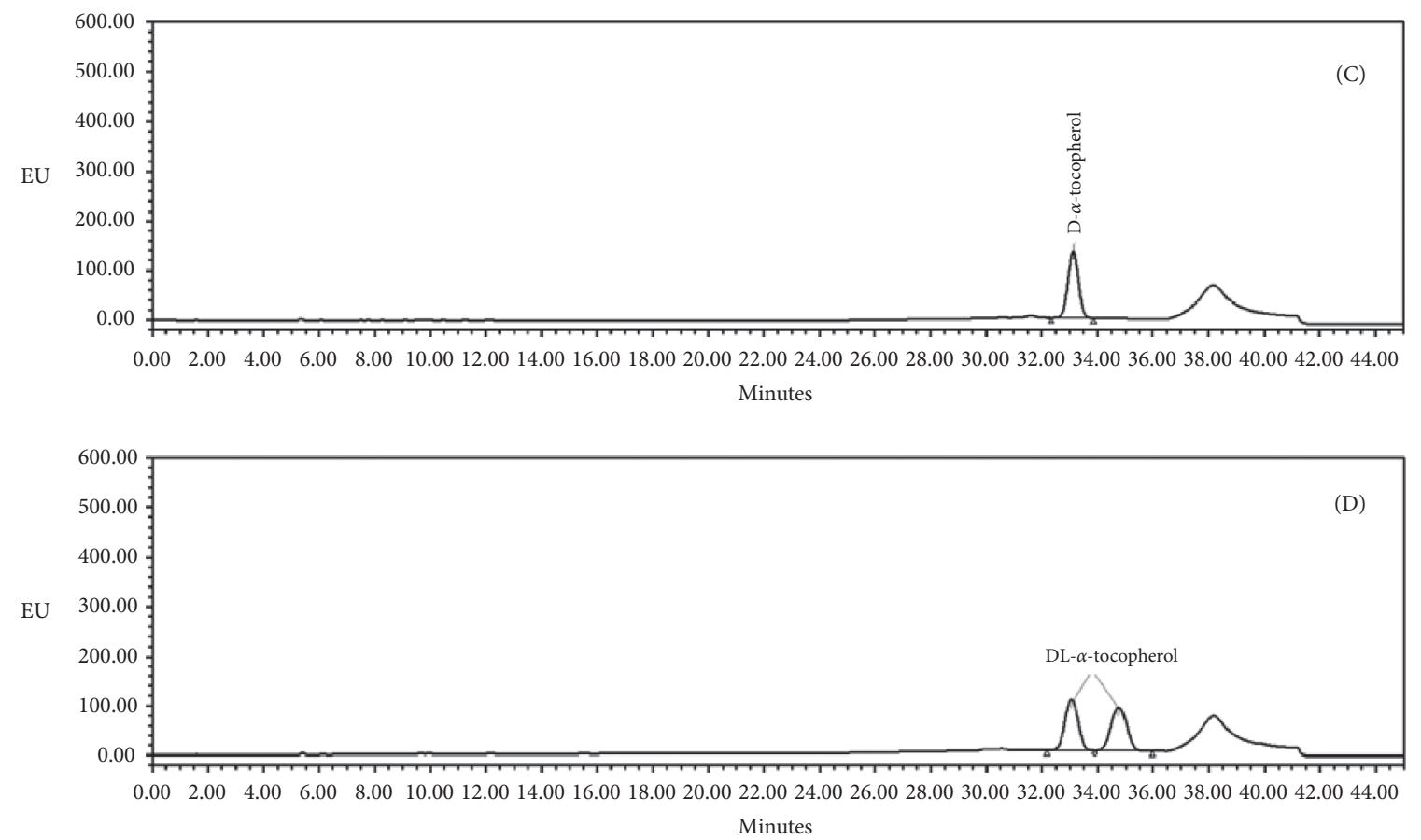

(c)
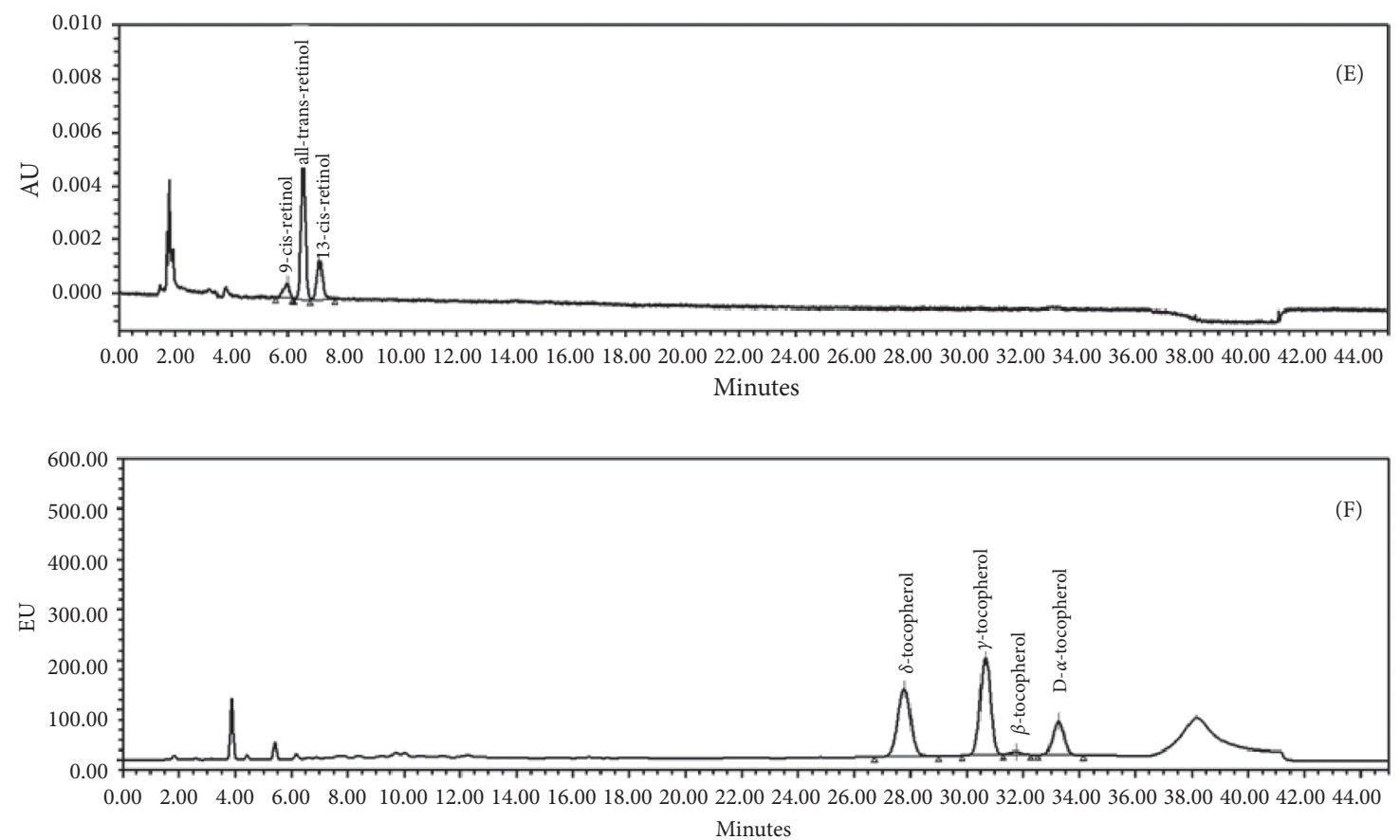

(d)

FIgURE 1: Typical chromatograms of (a) tocopherols and tocotrienols in fluorescent detector and (b) retinols in photo-array detector: (A) presents all analytes in mixture standard solution, (B) presents analytes in matrix extraction, (c) presents peaks of D- $\alpha$-tocopherol (C) and DL- $\alpha$-tocopherol (D) in the relative standard solution, (d) presents chromatograms of components in SRM 1849a, (E) retinols in photoarray detector, (F) tocopherols and tocotrienols in fluorescent detector.

$200 \mathrm{mg}$ size column. And the speed of extracts passed by Bond Elut Plexa column $(500 \mathrm{mg}, 6 \mathrm{~mL})$ was much slower during the loading step. Thus, both of SelectcCore PSN (200 mg, $6 \mathrm{~mL}$ ) and Welchrom PS/DVB (200 mg, $6 \mathrm{~mL}$ ) could be chosen in the following steps.
For cleaner samples and reducing ion suppression, the washing solution was optimized. Different percentages of organic solution $(10 \mathrm{~mL}$ of $0 \%, 2 \%, 5 \%, 10 \%$ methanol solution, and $40 \%$ ethanol, respectively) were compared and the results showed $10 \%$ methanol solution was the best with 


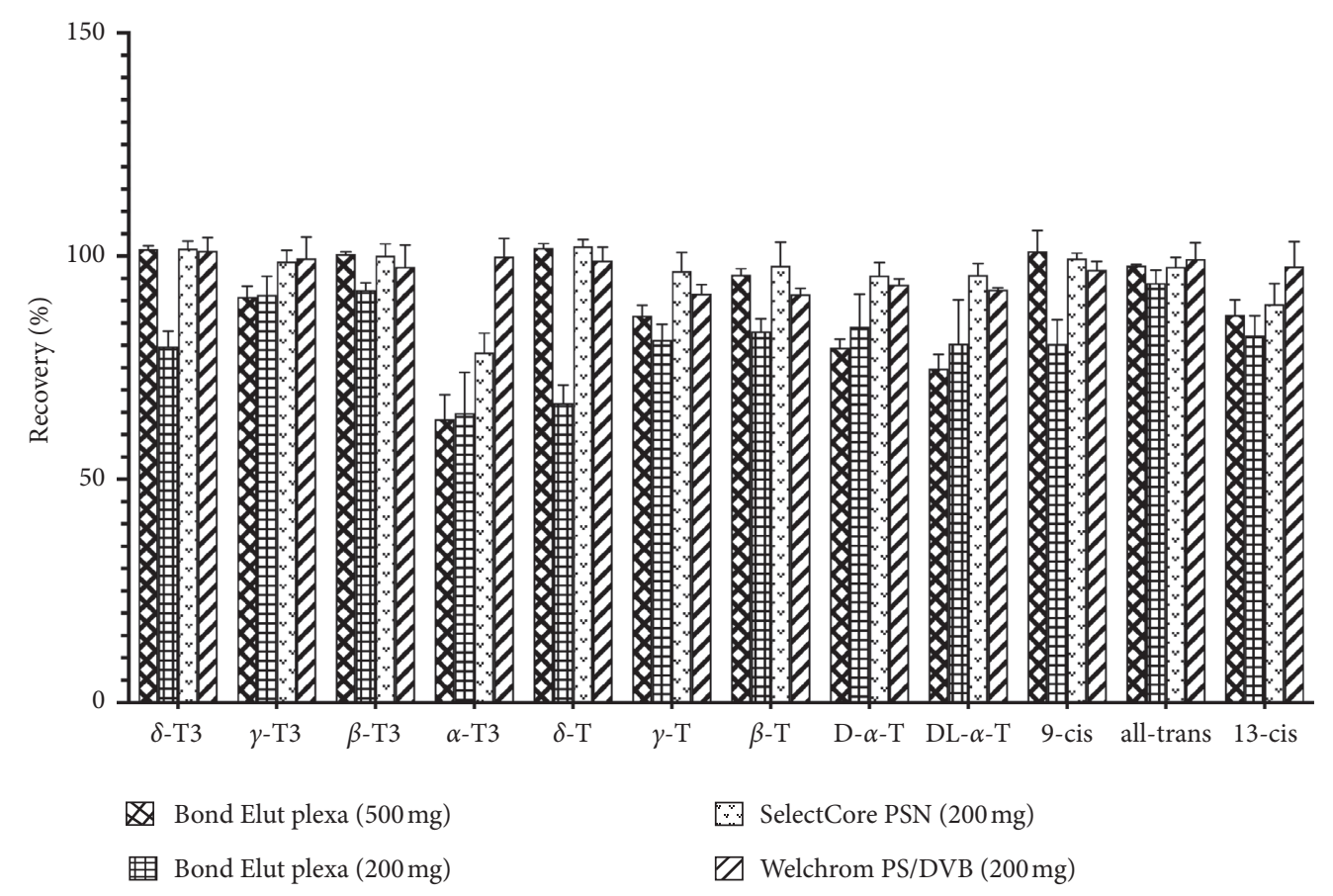

FIGURE 2: Appearances of different brand SPE columns on retinols, tocopherols, and tocotrienols (T: tocopherol; T3: tocotrienol; 9-cis: 9-cisretinol; 13-cis: 13-cis-retinol; all-trans: all-trans-retinol).

higher than $80 \%$ recoveries of all analytes. Recoveries of $\delta$-tocols were lower than $70 \%$ when $40 \%$ ethanol solution was used for washing, which explicated a higher organic washing solution was undesirable. In the elution step, $7.5 \mathrm{~mL}$ of $\mathrm{ACN} / \mathrm{MeOH}$ mixture $(75 / 25, \mathrm{v} / \mathrm{v})$ was necessary to provide high recoveries of analytes, which ranged from $90.25 \%$ to $103.3 \%$ for tocotrienols, $89.54 \%$ to $98.36 \%$ for tocopherols, and 93.13 to $116.03 \%$ for retinols.

Besides being satisfied with the simulated saponification solution, it would be available for real infant formula samples. To confirm the SPE conditions, a mixture of several different brands of infant formula samples was conducted for the sake of enriching tocols and retinols instead of spiking standards. Also, it is more economical and effective to investigate the purification ability and applicability of the SPE method to potential impurities. The results were evaluated by each compound content in the mixture sample. In practice, there was no difference in washing and eluting steps between simulation solution and mixture infant formula sample, except the loading step. When loading sample saponification extract directly, the contents of $\delta$-tocols would be half of that from the loading sample with a onefold volume of water. This is matched with the simulation extract recoveries obtained from $40 \%$ ethanol used as a washing solution. The high percentage of ethanol in saponification extract would cause less reservation of analytes. Onefold bulk of the water was added to decrease the percentage of organic solvent.

3.3. Analytical Characteristics of the Method. The typical chromatograms of analytes extracted from the mixture infant formula sample and standards solution are presented in Figures 1(a) and 1(b), as well as DL- $\alpha$-tocopherol and D- $\alpha$-tocopherol standard solution (Figure 1(c)). The separation of each retinol and tocol compound exhibited good specificity. No unidentified peaks in the selected samples interfered with the analytes.

The linearity calculation was based on the six increasing concentrations of the standard solution of each isomer. Ranging from $0.01 \mu \mathrm{g} \cdot \mathrm{mL}^{-1}$ to $25 \mu \mathrm{g} \cdot \mathrm{mL}^{-1}$, all of retinols and tocols compounds showed good linear regressions $(r>0.999)$ as displayed in Table 1, which allowed acquiring reliable and effective data for infant formula samples and relative modified products with low and high contents of vitamin $\mathrm{E}$ and retinols. The LODs and LOQs, from $0.05 \mathrm{mg} /$ $\mathrm{kg}$ to $0.4 \mathrm{mg} \cdot \mathrm{kg}^{-1}$, and from $0.15 \mathrm{mg} \cdot \mathrm{kg}^{-1}$ to $1.2 \mathrm{mg} \cdot \mathrm{kg}^{-1}$, respectively, were reported here matched with small amounts of analytes in infant formula samples, which referred to the sensitivity of the instrument.

Accuracy and precious constructed for the spiked infant formula sample with approximate standard concentration, prepared as described in Section 2.4, are presented in Table 2. It is noticed that the results performed excellent repeatability and satisfactory precision, with RSD values lower than $11 \%$, and mean recoveries were between $74.66 \%$ and $112.92 \%$.

Finally, the reliability of the method was further checked by using the reference material SRM 1849a. The results obtained are listed in Table 3 and illustrated in Figure 1(d). Figure 1(d) displays the variety of components in SRM 1849a, containing cis and trans retinols, and four isomers of tocopherols $(\alpha-, \beta-, \gamma$-, and $\delta$-), compared with NIST official document report. The form of $\alpha$-tocopherol is D- $\alpha$-tocopherol mainly, for the sake of one 
TABLE 1: Parameters of the RP-LC method for determination of vitamin E and retinol isomers.

\begin{tabular}{|c|c|c|c|c|c|c|}
\hline Analyte & $\mathrm{RT}(\mathrm{min})$ & Calibration curve & $r$ & LOD $\left(\mathrm{mg} \mathrm{kg}^{-1}\right)$ & LOQ $\left(\mathrm{mg} \mathrm{kg}^{-1}\right)$ & Range $\left(\mu \mathrm{g} \mathrm{mL}^{-1}\right)$ \\
\hline All-trans-retinol & 6.53 & $y=0.0042 x-3.00$ & 0.9999 & 0.05 & 0.15 & $0.1 \sim 5$ \\
\hline 13-cis-retinol & 7.09 & $y=0.0046 x-0.36$ & 1.0000 & 0.05 & 0.15 & $0.01 \sim 0.5$ \\
\hline 9-cis-retinol & 5.97 & $y=0.0041 x-1.43$ & 1.0000 & 0.05 & 0.15 & $0.01 \sim 0.5$ \\
\hline DL- $\alpha$-tocopherol ${ }^{(a)}$ & $33.34 / 35.10$ & $y=0.0006 x-74.28$ & 0.9998 & 0.4 & 1.2 & $2 \sim 100$ \\
\hline D- $\alpha$-tocopherol & 33.34 & $y=0.0006 x-119.01$ & 0.9998 & 0.4 & 1.2 & $0.5 \sim 25$ \\
\hline $\mathrm{D}-\beta$-tocopherol & 31.93 & $y=0.0002 x-10.63$ & 0.9999 & 0.4 & 1.2 & $0.5 \sim 25$ \\
\hline$D-\gamma$-tocopherol & 30.84 & $y=0.0001 x+49.20$ & 0.9999 & 0.4 & 1.2 & $0.5 \sim 25$ \\
\hline$D$ - $\delta$-tocopherol & 27.99 & $y=0.0001 x+10.72$ & 0.9999 & 0.4 & 1.2 & $0.5 \sim 25$ \\
\hline $\mathrm{D}-\alpha$-tocotrienol & 26.92 & $y=0.0006 x+108.41$ & 0.9999 & 0.4 & 1.2 & $0.5 \sim 25$ \\
\hline$D$ - $\beta$-tocotrienol & 24.32 & $y=0.0002 x+7.62$ & 0.9996 & 0.4 & 1.2 & $0.5 \sim 25$ \\
\hline D- $\gamma$-tocotrienol & 22.29 & $y=0.0002 x+47.58$ & 0.9999 & 0.4 & 1.2 & $0.5 \sim 25$ \\
\hline $\mathrm{D}$ - $\delta$-tocotrienol & 18.30 & $y=0.0001 x-12.00$ & 0.9999 & 0.4 & 1.2 & $0.5 \sim 25$ \\
\hline
\end{tabular}

(a) Two ideal peaks appeared in retention time (RT) of $33.34 \mathrm{~min}$ and $35.10 \mathrm{~min}$, within the approximate peak area.

TABLE 2: Accuracy and precious in spiked infant formula samples $(n=6)$.

\begin{tabular}{|c|c|c|c|c|c|c|c|}
\hline \multirow{2}{*}{ Analyte } & \multirow{2}{*}{$\operatorname{Blank}^{(\mathrm{a})}\left(\mathrm{mg} \mathrm{kg}^{-1}\right)$} & \multicolumn{2}{|c|}{ Spiking level $1^{(b)}$} & \multicolumn{2}{|c|}{ Spiking level 2} & \multicolumn{2}{|c|}{ Spiking level 3} \\
\hline & & Recovery (SD) \% & $\mathrm{RSD} \%$ & Recovery (SD) \% & $\mathrm{RSD} \%$ & Recovery (SD) \% & RSD $\%$ \\
\hline All-trans-retinol & $2.25(0.10)$ & $95.31(3.46)$ & 3.63 & $82.49(3.18)$ & 3.85 & $82.54(3.21)$ & 3.89 \\
\hline 13-cis-retinol & $0.74(0.10)$ & $74.66(2.72)$ & 3.64 & $95.12(9.80)$ & 10.30 & $98.63(9.61)$ & 9.74 \\
\hline 9-cis-retinol & $0.39(0.10)$ & $112.92(5.71)$ & 5.05 & $96.40(6.27)$ & 6.50 & $97.23(4.38)$ & 4.51 \\
\hline DL- $\alpha$-tocopherol & $114.19(2.02)$ & $103.22(4.19)$ & 3.9 & 91.34 (2.59) & 2.86 & $85.70(1.07)$ & 1.25 \\
\hline D- $\alpha$-tocopherol & $69.85(2.33)$ & $104.12(6.20)$ & 5.68 & $93.35(2.65)$ & 2.84 & $87.24(0.84)$ & 0.97 \\
\hline D- $\beta$-tocopherol & $1.26(0.15)$ & $92.22(1.41)$ & 1.53 & $89.29(1.49)$ & 1.67 & $91.62(0.59)$ & 0.65 \\
\hline D- $\gamma$-tocopherol & $23.14(0.92)$ & $92.70(9.75)$ & 10.52 & $90.93(5.28)$ & 5.81 & $90.90(3.57)$ & 3.92 \\
\hline $\mathrm{D}$ - $\delta$-tocopherol & $10.42(0.47)$ & $81.15(6.67)$ & 8.22 & $84.14(4.28)$ & 5.08 & $83.16(2.24)$ & 2.69 \\
\hline D- $\alpha$-tocotrienol & $2.42(0.00)$ & $76.38(4.03)$ & 5.28 & $90.31(2.69)$ & 2.97 & $89.53(2.70)$ & 3.02 \\
\hline $\mathrm{D}-\beta$-tocotrienol & ND & $109.38(9.95)$ & 9.10 & $97.18(4.21)$ & 4.33 & $95.35(2.90)$ & 3.05 \\
\hline D- $\gamma$-tocotrienol & $1.07(0.00)$ & $79.81(0.16)$ & 0.20 & $82.03(4.49)$ & 5.47 & $83.83(1.90)$ & 2.27 \\
\hline $\mathrm{D}-\delta$-tocotrienol & $0.78(0.00)$ & $82.98(3.38)$ & 4.07 & $85.15(1.00)$ & 1.18 & $85.92(1.16)$ & 1.35 \\
\hline
\end{tabular}

${ }^{(a)} \mathrm{ND}$ represents mass fraction of the analyte in sample was lower than LOD. ${ }^{(\mathrm{b})}$ Spiking concentrations were based 0.75 -, 1.5-, and 3-folds on the content of analytes in infant formula sample, which was mainly calculated based on the content of D- $\alpha$-tocopherol.

TABLE 3: Retinol and vitamin E isomer contents in certified reference materials (SRM 1849a).

\begin{tabular}{|c|c|c|c|c|c|c|c|c|}
\hline & \multicolumn{4}{|c|}{ Vitamin A $\left(\mathrm{mg} \mathrm{kg}^{-1}\right)$} & \multicolumn{4}{|c|}{ Vitamin E $\left(\mathrm{mg} \mathrm{kg}^{-1}\right)^{(\mathrm{b})}$} \\
\hline & All-trans-retinol & 13-cis-retinol & 9-cis-retinol & Total $^{(a)}$ & D- $\alpha$-tocopherol & $\beta$-tocopherol & $\gamma$-tocopherol & $\delta$-tocopherol \\
\hline Content & $5.91 \pm 0.13$ & $1.80 \pm 0.05$ & $0.81 \pm 0.04$ & $7.71 \pm 0.14$ & $221 \pm 4$ & $5.59 \pm 0.36$ & $141 \pm 4$ & $76.1 \pm 2.1$ \\
\hline Certified value & \multicolumn{4}{|c|}{$\begin{array}{l}\left.7.68 \pm 0.23 \mathrm{mg} \cdot \mathrm{kg}^{-1} \text { retinol equivalents, total (cis }+ \text { trans }\right) \\
\text { retinol without any biological activity correction }\end{array}$} & \multicolumn{4}{|c|}{$\begin{array}{c}219 \pm 16 \mathrm{mg} \cdot \mathrm{kg}^{-1} \alpha \text {-tocopherol equivalents, including natural } \\
\alpha \text {-tocopherol and added } \alpha \text {-tocopheryl acetate }\end{array}$} \\
\hline
\end{tabular}

peak, appeared in relative retention time. Table 3 shows the mass fraction of total (cis and trans) retinols was a little bit higher than the certified content, whereas the total of all-trans and 13-cis retinols was in agreement. With respect to vitamin $\mathrm{E}$, the result of $\mathrm{D}$ - $\alpha$-tocopherol was in the range of assigned value, and the contents of $\gamma$ and $\delta$-tocopherols were also abundant. Such data confirmed the efficacy of the methodology and the extraction procedure. And what is more, it is necessary to identify each isomer of retinols and tocols compound in infant formula when evaluating biological activity and estimate the equivalent by rule and line.

\section{Concluding Remarks}

The optimized RP-LC method offers advantages over previous literature, such as simultaneous quantitation of variety analytes, quicker distinction of $\alpha$-tocopherol form, and estimation of common retinol isomers. The choice chiral chromatographic column was recommended to utilize in routine practice for the relatively low cost and available effective time. For the trend of quicker, simpler, cheaper, rugger, and safer requests in sample preparations, the SPE method takes place of the conventional solvent extraction method. Although the sorbent (PS-DVB) in the packing 
column is not a novel material, it is the first time to be employed in vitamin $\mathrm{E}$ and retinols concentrated.

\section{Abbreviations}

NP-LC: Normal-phase liquid chromatography

RP-LC: Reverse-phase liquid chromatography

PFP: $\quad$ Pentafluorophenyl

SPE: $\quad$ Solid-phase extraction

MeOH: Methanol

ACN: Acetonitrile

LOD: Limits of detection

LOQ: $\quad$ Limits of detection

S/N: $\quad$ Signal-to-noise ratio

BHT: Butylated hydroxytoluene

PS-DVB: Polystyrene divinylbenzene.

\section{Data Availability}

The data used to support the study are included within the article.

\section{Conflicts of Interest}

The authors declare no conflicts of interest.

\section{Acknowledgments}

This research did not receive any specific grant from funding agencies in the public, commercial, or not-for-profit sectors.

\section{References}

[1] O. A. Roels and J. P. Mack, "Vitamin A and protein metabolism," Journal of Agricultural and Food Chemistry, vol. 20, no. 6, pp. 1133-1135, 1972.

[2] M. L. Colombo, "An update on vitamin E, tocopherol and tocotrienol-perspectives," Molecules, vol. 15, no. 4, pp. 2103-2113, 2010.

[3] H. Weiser and G. Somorjai, "Bioactivity of cis and dicis isomers of vitamin A esters," International Journal for Vitamin and Nutrition Research, vol. 62, no. 3, pp. 201-208, 1992.

[4] Y. Yui, S. Miyazaki, Y. Ma et al., "Distinction of synthetic dl$\alpha$-tocopherol from natural vitamin E (d- $\alpha$-tocopherol) by reversed-phase liquid chromatography. enhanced selectivity of a polymeric C18 stationary phase at low temperature and/ or at high pressure," Journal of Chromatography A, vol. 1450, pp. 45-52, 2016.

[5] F. J. Ruperez, D. Martin, E. Herrera, and C. Barbas, "Chromatographic analysis of a-tocopherol and related compounds in various matrices," Journal of Chromatography A, vol. 935, no. 1-2, pp. 45-69, 2001.

[6] H. Scherf, L. J. Machlin, T. M. Frye, B. A. Krautmann, and S. N. Williams, "Vitamin E biopotency: comparison of various "natural-derived" and chemically synthesized $\alpha$-tocopherols," Animal Feed Science and Technology, vol. 59, no. 1-3, pp. 115-126, 1996.

[7] M. Miyagi, H. Yokoyama, H. Shiraishi, M. Matsumoto, and H. Ishii, "Simultaneous quantification of retinol, retinal, and retinoic acid isomers by high-performance liquid chromatography with a simple gradiation," Journal of
Chromatography B: Biomedical Sciences and Applications, vol. 757, no. 2, pp. 365-368, 2001.

[8] R. M. D. Fávaro, M. H. Iha, T. C. Mazzi, R. Fávaro, and M. de Lourdes Pires Bianchi, "Stability of vitamin A during storage of enteral feeding formulas," Food Chemistry, vol. 126, pp. 827-830, 2011.

[9] P. Viñas, M. Bravo-Bravo, I. López-García, and M. Hernández-Córdoba, "An evaluation of cis- and transretinol contents in juices using dispersive liquid-liquid microextraction coupled to liquid chromatography with fluorimetric detection," Talanta, vol. 103, pp. 166-171, 2013.

[10] C. Fanali, G. D’Orazio, S. Fanali, and A. Gentili, "Advanced analytical techniques for fat-soluble vitamin analysis," TrAC Trends in Analytical Chemistry, vol. 87, pp. 82-97, 2017.

[11] W. Stöggl, C. Huck, S. Wongyai, H. Scherz, and G. Bonn, "Simultaneous determination of carotenoids, tocopherols, and $\gamma$-oryzanol in crude rice bran oil by liquid chromatography coupled to diode array and mass spectrometric detection employing silica C30 stationary phases," Journal of Separation Science, vol. 28, no. 14, pp. 1712-1718, 2005.

[12] R. K. Saini and Y.-S. Keum, "Tocopherols and tocotrienols in plants and their products: a review on methods of extraction, chromatographic separation, and detection," Food Research International, vol. 82, pp. 59-70, 2016.

[13] N. E. Craft, Tocopherols: Properties and Determination, Elsevier, Amsterdam, Netherlands, 1st edition, 2016.

[14] T. Ueda, H. Ichikawa, and O. Igarashi, "Determination of alpha-tocopherol stereoisomers in biological specimens using chiral phase high-performance liquid chromatography," Journal of Nutritional Science and Vitaminology, vol. 39, no. 3, pp. 207-219, 1993.

[15] CEN 12823-1:2014, Foodstuffs-Determination of Vitamin A by High Performance Liquid Chromatography Part I: Measurement of All-trans Retinol and 13-cis Retinol.

[16] CEN 12822:2014, Foodstuffs-Determination of vitamin E by high performance liquid-Measurement of $\alpha_{-}^{-}, \beta_{-}, \gamma^{-}$, and $\delta$-tocopherols.

[17] National Health and Family Planning Commission of the People's Republic of China, GB 5009.82-2016 Foodstuffs safety mandatory standard Determination of vitamin A/D/E in food.

[18] T.-S. Shin, J. S. Godber, D. E. Martin, and J. H. Wells, "Hydrolytic stability and changes in E vitamers and oryzanol of extruded rice bran during storage," Journal of Food Science, vol. 62, pp. 704-728, 1997.

[19] M. N. Irakli, V. F. Samanidou, and I. N. Papadoyannis, "Development and validation of an HPLC method for the simultaneous determination of tocopherols, tocotrienols and carotenoids in cereals after solid-phase extraction," Journal of Separation Science, vol. 34, no. 12, pp. 1375-1382, 2011.

[20] M. Podda, C. Weber, M. G. Traber, and L. Packer, "Simultaneous determination of tissue tocopherols, tocotrienols, ubiquinols, and ubiquinones," Journal of Lipid Research, vol. 37, no. 4, pp. 893-901, 1996.

[21] A. McMahon, "Determination of vitamin E and vitamin A in infant formula and adult nutritionals by normal-phase high-performance liquid chromatography: collaborative study, final action 2012.10," Journal of AOAC International, vol. 99, pp. 223-241, 2016. 